\title{
A Balanced-to-Balanced In-Phase Filtering Power Divider (FPD) With High Selectivity and Isolation
}

\author{
Feng Wei, Member, IEEE, Zhi-Jie Yang, Pei-Yuan Qin, Member, IEEE, Y. Jay Guo, Fellow, IEEE, Bin \\ Li, Member, IEEE, and Xiao-Wei Shi, Senior Member, IEEE
}

\begin{abstract}
In this paper, a balanced-to-balanced (BTB) inphase filtering power divider (FPD) is proposed, which can realize a two-way equal power division with high selectivity and isolation. The design of the proposed FPD is primarily based on microstrip/slotline transition structures and slotline T-junction. A U-type microstrip feed line integrated with a stepped-impedance slotline resonator is adopted at the input and output ports, which makes the differential-mode (DM) responses independent of the common-mode (CM) ones. Meanwhile, superior DM transmission and $C M$ suppression are achieved intrinsically, thereby simplifying the design procedure significantly. By employing slotline resonators loaded with resistors, the isolation between the two output ports can be improved greatly. In addition, a DM passband with a sharp filtering performance is realized by introducing the microstrip stub-loaded resonators (SLRs). By changing the electrical length of the open-stub of the SLR, the fractional bandwidth (FBW) is controllable. In order to verify the feasibility of the proposed design method, two prototype circuits of the proposed FPDs with different bandwidths are fabricated and measured. Good agreement between the simulation and measurement results is observed.
\end{abstract}

Index Terms-Balanced-to-balanced (BTB), common-mode (CM) suppression, filtering power divider (FPD), high selectivity, isolation, stub-loaded resonator (SLR)

\section{INTRODUCTION}

$\mathrm{I}_{\mathrm{e}}^{\mathrm{N}}$ NECENT years, with the rapid development of high-speed Ielectronic systems, balanced/differential circuits have been utilized more and more widely and have attracted substantial attention due to their excellent performance in terms of the

This work was supported by the National Natural Science Foundation of China (NSFC) (grant 61771055), the Key Laboratory for Research of Design and Electromagnetic Compatibility of High Speed Electronic Systems, Ministry of Education, Basic Research Foundation of Beijing Institute of Technology (grant 20170542009), the Fundamental Research Funds for the Central Universities (grant JB180204) and State Key Laboratory of Metamaterial Electromagnetic Modulation Technology.

Feng Wei, Zhi-Jie Yang and Xiao-Wei Shi are with National Key Laboratory of Antennas and Microwave Technology and Collaborative Innovation Center of Information Sensing and Understanding at Xidian University, School of Electronic Engineering, Xidian University, Xi' an 710071 China (e-mail: fwei@mail.xidian.edu.cn).

Y. Jay Guo and Pei-Yuan Qin are with University of Technology Sydney, Global Big Data Technologies Center, Sydney, Australia.

$\mathrm{Bin} \mathrm{Li}$ is with the School of Information and Electronics, Beijing Institute of Technology, Beijing. immunity against environmental noises and electromagnetic interferences, compared with the single-end counterparts. In order to combine the balanced active circuits and differentially driven antennas to build up fully balanced transceivers, various balanced passive components have been proposed, including balanced-to-balanced (BTB) filters/power dividers (PDs). These devices can select the differential signals in the frequency domain and suppress the common-mode (CM) noise simultaneously. In the past ten years, many balancedto-balanced bandpass filters (BPFs) with single band and multiple bands have been realized [1]-[5]. Meanwhile, several balanced-to-balanced power dividers with good performance have been realized. In [6], a novel wideband balancedto-balanced network with unequal power division and wideband common mode suppression was proposed. A balanced-to-balanced power divider with arbitrary power division was presented in [7] for the first time. The application of the slotline structure has always been a hot topic in the research field of single-ended and balanced passive components [8]-[11]. Recently, several balanced-to-singleended and balanced-to-balanced power dividers employing slotlines were proposed successively. By etching both the slot and the aperture on the common ground of the back-to-back microstrip Wilkinson PD, a wideband balanced-to-balanced PD with a high CM suppression can be achieved [12]. Coincidentally, a wideband differential power dividing/ combining network with high isolation was designed and proposed in [13]. A compact differential PD using coupled microstrip line was presented in [14], which can divide the input differential-mode power into two ways with in-phase response or out-of-phase one. Moreover, an approach to 1-to- $2^{\mathrm{n}}$ way microstrip balanced power divider was proposed for the first time in [15].

When considering the practical application in whole balanced system, at least one balanced-to-balanced filter should be added before the balanced-to-balanced power divider if the enhancement of frequency selectivity is demanded, as shown in Fig. 1(a). In order to achieve miniaturization, low loss and low cost, dual-function components that can achieve both filtering and power dividing are emerging. Three novel wideband balanced-to-single-ended filtering power dividers (FPDs) using coupled lines were proposed in [16]. A balanced-to-unbalanced PD based on microstrip branch lines with several stubs and a 


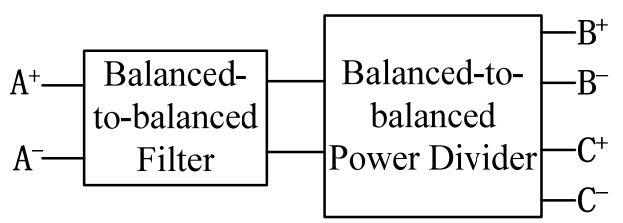

(a)

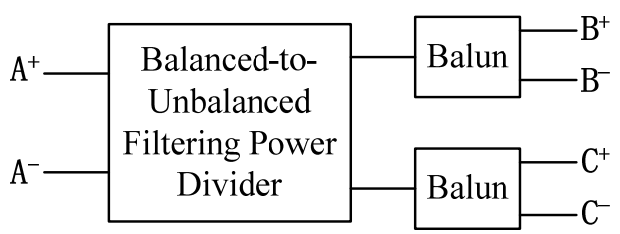

(b)

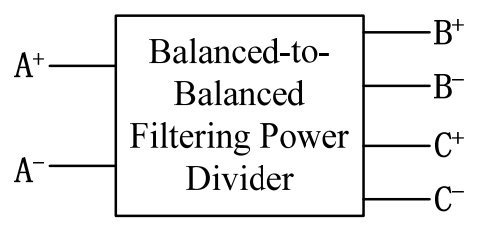

(c)

Fig. 1. (a) Cascade of a balanced filter and a balanced power divider, (b) Cascade of a balanced-to-unbalanced filtering power divider and two baluns, and (c) Balanced-to-balanced filtering power divider.

dual-band balanced-to-single-ended FPD by coupled ring resonators were proposed in [17] and [18], respectively. For the whole balanced system, at least two baluns are needed after the balanced-to-unbalanced FPDs, as shown in Fig.1(b). Therefore, balanced-to-balanced FPDs, as shown in Fig. 1(c), have more advantages in terms of the miniaturization and low loss. Up to now, only a few balanced-to-balanced FPDs have been proposed. In 2013, a balanced-to-balanced Gysel FPD was proposed for the first time [19], which has a CM suppression of about $20 \mathrm{~dB}$. One transmission zero is realized for improving the selectivity of the passband. Lately, another balancedto-balanced FPD using $\lambda_{g} / 2$ resonators and short-stub-loaded resonator was presented in [20], with an IL of $2.21 \mathrm{~dB}$ (excluding the $3 \mathrm{~dB}$ power division intrinsic loss). A simple FPD based on three coupled resonators for differential input and output signals was proposed in [21], which has an IL of 3.6 $\mathrm{dB}$ (excluding the $3 \mathrm{~dB}$ power division intrinsic loss) and a DM isolation of $9.1 \mathrm{~dB}$. In addition, a novel narrow-band balanced filtering network using coupled lines loaded with stubs was proposed in [22], which achieving a wideband CM suppression and isolation.

In this paper, a novel two-way equal balanced-to-balanced in-phase FPD is proposed and analyzed. The FPD is fed by balanced U-type stepped-impedance microstrip/slotline transition structures, which achieves an independent CM response with a larger bandwidth and a superior suppression. Since all the CM signals are intrinsically blocked, only its passband performance under the differential-mode (DM) operation needs to be investigated in the design process, which simplifies the design procedure significantly. A uniform impedance slotline T-junction is designed to equal power division. Meanwhile, two stub-loaded resonators (SLRs) are introduced to achieve a sharp passband with a controllable fractional bandwidth (FBW). Furthermore, a novel and simple

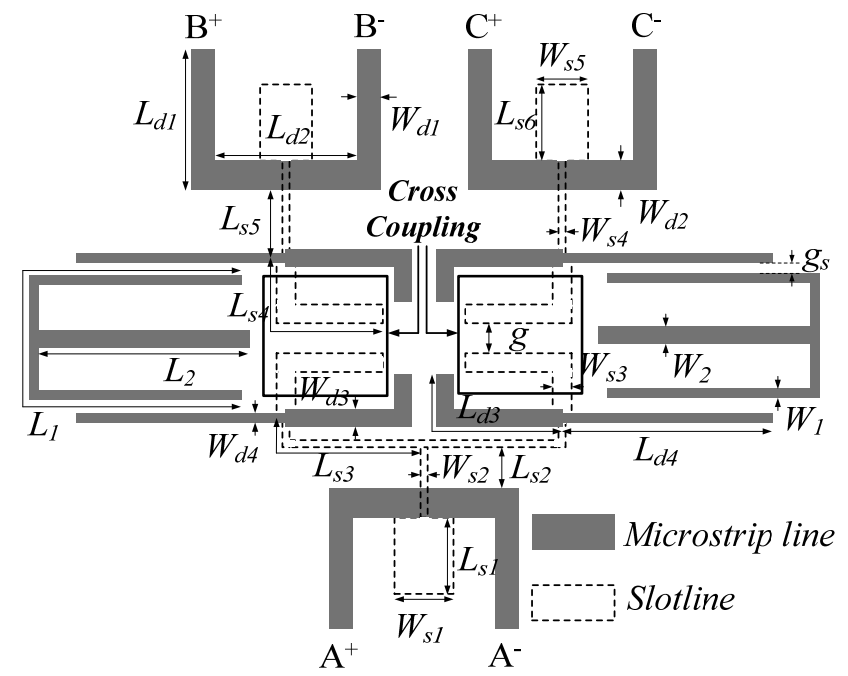

Fig. 2. Configuration of the proposed FPD without isolation resistors.

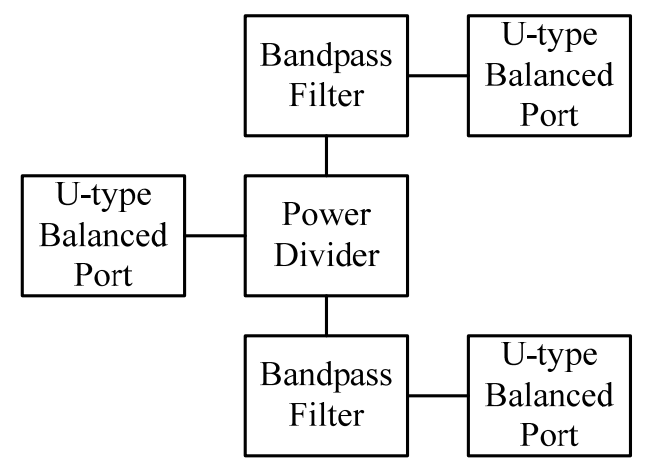

Fig. 3. The internal schematic illustration of the proposed FPD

approach is proposed to improve the DM isolation between the two output ports greatly. In the end, simplified equivalent circuits are derived and synthesized.

Compared to other reported work, the contributions of the proposed FPD are follows. Firstly, a completed new structure is proposed for balanced-to-balanced FPD. Secondly, overall superior performance has been achieved over many reported designs. For example, there are two TZs on both sides of the passband to enhance the selectivity greatly. The maximum in-band IL is $0.8 \mathrm{~dB}$. The bandwidth of the CM response is broad with a more than $45 \mathrm{~dB} C M$ suppression and a more than $18 \mathrm{~dB}$ DM isolation. Thirdly, the proposed structure has a great flexibility to change the center frequencies and the bandwidths to meet different system requirements. This is evidenced by the two prototypes designed and fabricated with different operating bandwidths.

The rest of the paper is organized as follows. The performance of the proposed FPD without isolation resistors is analyzed in Section II, and the phase analysis and DM equivalent circuit analysis of the proposed FPD with high isolation performance are presented in Section III. Then, the simulated and measured results of the proposed FPD with high isolation performance are shown in Section IV. Section V concludes the paper. 


\section{BALANCED-TO-BALANCED FILTERING POWER DIVIDER}

\section{A. Overall Structure and Design Principle}

A slotline is a planar transmission structure proposed by Cohn in 1968, which consists of a narrow slot etched in the metallization on one side of a dielectric substrate. The metal on the other side of the substrate is removed. This geometry is well suited for applications in microwave intergrated circuits.

The configuration of the proposed FPD without isolation resistors is shown in Fig. 2. It is composed of balanced input/output U-type stepped-impedance microstrip/slotline transition structures (will be studied in sub-section B), slotline T-junction and microstrip SLRs (will be studied in sub-section C). Fig. 3 shows the internal schematics of the proposed FPD. It can be seen that the design principle of the proposed balanced-to-balanced FPD can be simply viewed as a power divider cascaded with two bandpass filters (BPFs).

Similar to [23], transmission-line equivalent circuit of the proposed FPD under DM operation can be obtained as presented in Fig. 4, where $Z_{n}(n=0,1,2,3)$ and $Z_{s n}(n=$ $1,2,3,4,5,6)$ are the microstrip line and slotline characteristic impedances, respectively. Meanwhile, $Z_{e}$ and $Z_{o}$ are even-mode and odd-mode equivalent impedances of the parallel coupled microstrip lines, respectively. The electric lengths of the evenand odd-mode, which are marked as $\theta$, can be assumed as equal to simplify the calculation and analysis process. $\theta_{1}=2 \pi l_{1} / \lambda_{1},\left(\lambda_{1}\right.$ is the guided wavelength of microstrip line at the center frequency of the proposed FPD) and $\theta_{s n}=2 \pi l_{s n} / \lambda_{s n}(n=1,4,6)$ $\left(\lambda_{s n}\right.$ is the guided wavelength of slotline at the center frequency of the proposed FPD) denote the microstrip line electrical length of the extended portion and the slotline electrical lengths of the extended portions, respectively. The microstrip/slotline transition structure can be expressed as an equivalent transformer. The transformer turn ratio $n$ represents the coupling magnitude between the microstrip line and slotline. In the analysis, a closed-form expression for the transformer turn ratio $n$ is given as [24]:

$$
\begin{aligned}
& n=\frac{J_{0}\left(k_{e s} W_{m} / 2\right) J_{0}\left(k_{e m} W_{s} / 2\right)}{k_{e s}^{2}+k_{e m}^{2}} \\
& \cdot\left[\frac{k_{e m}^{2} k_{2} \varepsilon_{r}}{k_{2} \varepsilon_{r} \cos k_{1} h-k_{1} \sin k_{1} h}+\frac{k_{e s}^{2} k_{1}}{k_{1} \varepsilon_{r} \cos k_{1} h-k_{2} \sin k_{1} h}\right]
\end{aligned}
$$

where $J_{0}(\cdot)$ is the zeroth-order Bessel function and

$$
\begin{aligned}
& k_{1}=\sqrt{\left|k_{0}^{2} \varepsilon_{r}-k_{e s}^{2}-k_{e m}^{2}\right|}=k_{0} \sqrt{\left|\varepsilon_{r}-\varepsilon_{r e s}-\varepsilon_{r e m}\right|} \\
& k_{2}=k_{0} \sqrt{\left|\varepsilon_{r e s}+\varepsilon_{r e m}-1\right|} \\
& k_{e s}=k_{0} \sqrt{\varepsilon_{r e s}} \\
& k_{e m}=k_{0} \sqrt{\varepsilon_{r e m}}
\end{aligned}
$$

where, $\varepsilon_{\text {rem }}$ and $\varepsilon_{\text {res }}$ are the effective dielectric constants of the microstrip line and the slotline, respectively.

According to the equivalent circuit (shown in Fig. 4) of the proposed BTB FPD, the reflection and transmission coefficients of the proposed BTB FPD can be calculated according to its equivalent circuit. Therefore, its frequency response can also be analyzed and optimized. Fig. 5 shows the comparison of the calculated frequency response from its

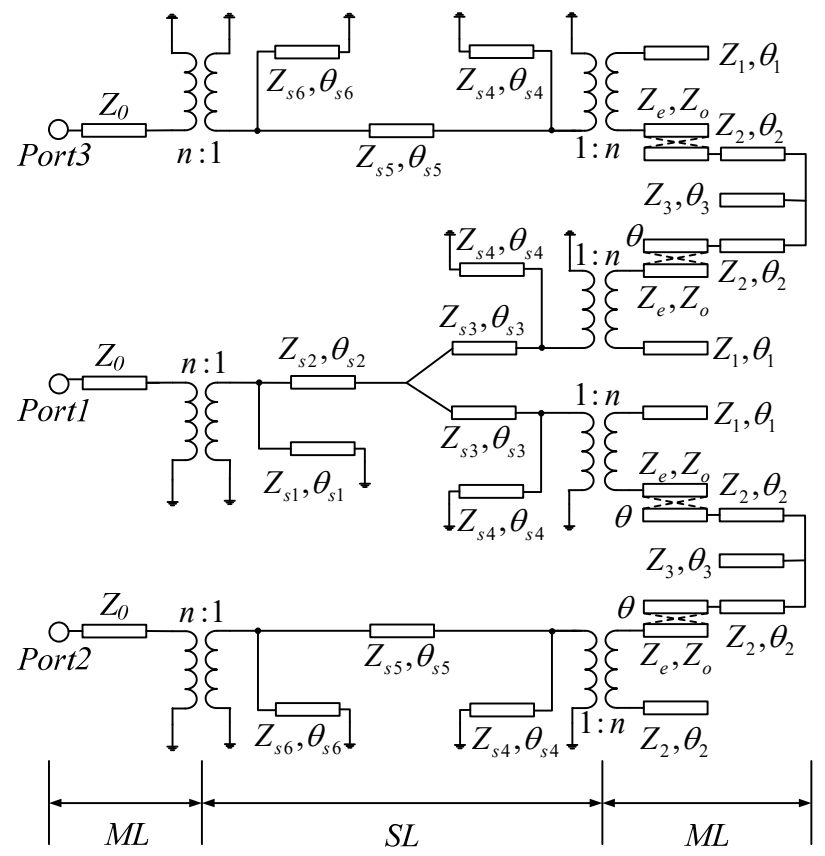

Fig. 4. The transmission-line equivalent circuit of the proposed FPD under $\mathrm{DM}$ operation. $(\mathrm{ML}=$ Microstrip Line; $\mathrm{SL}=$ Slotline $)$

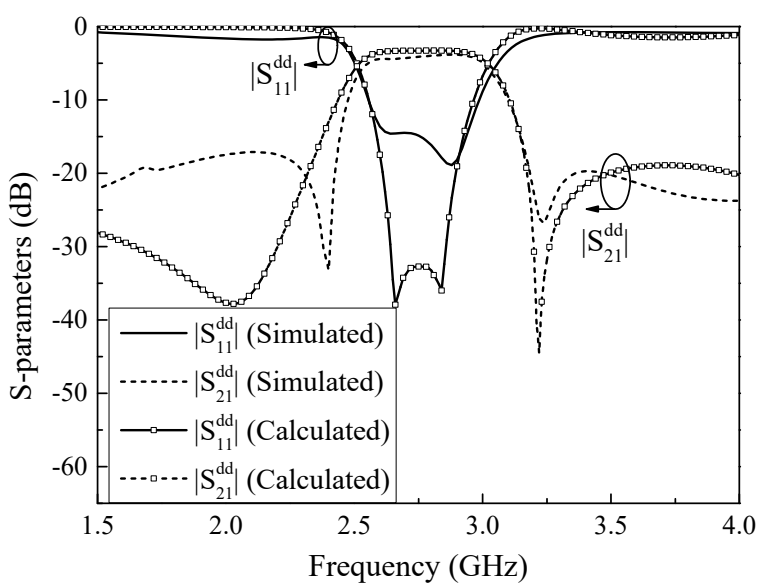

Fig. 5. Calculated and simulated frequency responses of the proposed BTB FPD. $\left(W_{d 1}=2.4 \mathrm{~mm}, W_{d 2}=3.0 \mathrm{~mm}, W_{d 3}=1.2 \mathrm{~mm}, W_{d 4}=0.5 \mathrm{~mm}, W_{l}=0.5\right.$ $\mathrm{mm}, W_{2}=1.2 \mathrm{~mm}, W_{s 1}=6.0 \mathrm{~mm}, W_{s 2}=0.2 \mathrm{~mm}, W_{s 3}=1.0 \mathrm{~mm}, W_{s 4}=0.2 \mathrm{~mm}$, $W_{s 5}=5.0 \mathrm{~mm}, W_{i}=0.2 \mathrm{~mm}, L_{d 1}=15 \mathrm{~mm}, L_{d 2}=14.4 \mathrm{~mm}, L_{d 3}=12.4 \mathrm{~mm}, L_{d 4}=$ $16.0 \mathrm{~mm}, L_{1}=42.0 \mathrm{~mm}, L_{2}=17.0 \mathrm{~mm}, L_{s l}=8.0 \mathrm{~mm}, L_{s 2}=3.0 \mathrm{~mm}, L_{s 3}=12.2$ $\left.\mathrm{mm}, L_{s 4}=13.5 \mathrm{~mm}, L_{s 5}=5.0 \mathrm{~mm}, L_{s 6}=7.5 \mathrm{~mm}, g_{s}=0.3 \mathrm{~mm}, g=2 \mathrm{~mm}\right)$

equivalent circuit with the simulated one by using an electromagnetic simulation software High Frequency Structure Simulator (HFSS). The frequency response coming from the equivalent circuits is calculated by Advanced Design System (ADS). The position of the first transmission zero (TZ) is directly related to the strength of cross coupling between the slotlines. In ADS, all slotlines have to be modeled as microstrip transmission lines, which makes the position of the first TZ different from that calculated by full-wave simulator HFSS as given in Fig. 5. But, a reasonably good agreement is achieved between the two responses, which validates the equivalent circuit. The optimized dimensions of the proposed BTB FPD can be obtained under the desired frequency response.

In differential systems, complementary signals (those of the 


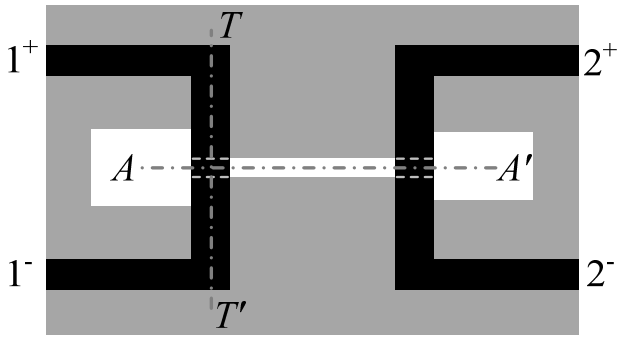

Fig. 6. Configuration of the balanced microstrip/slotline transition structure.

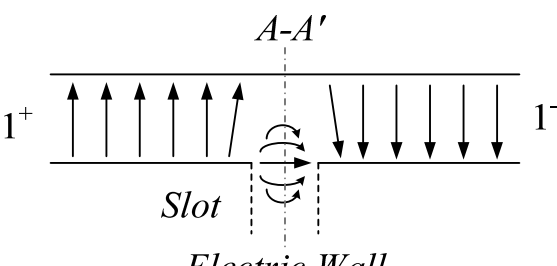

(a)

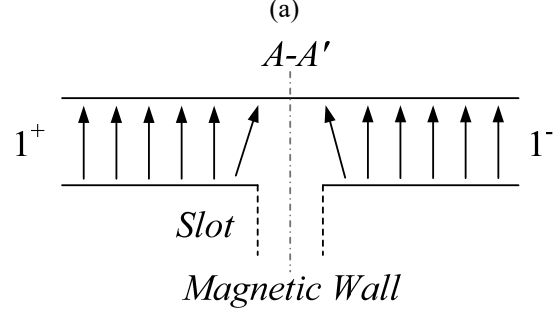

(b)

Fig. 7. The cross-views of electrical field distribution at $T-T^{\prime}$ plane. (a) Under DM operation. (b) Under CM operation.

same amplitude with a $180^{\circ}$ phase difference) are transmitted by a pair of terminals. And $S^{d d}$ represents the DM response and stimulus, and $S^{c c}$ represents the CM response and stimulus. In this case, $S_{I l}^{\text {ld }}$ is the return loss, and $S_{2 l}^{d d}$ is the insertion loss under DM operation. When the RF signals are excited at the balanced input port, the DM signals will be transmitted to the slotline and be transferred to the slotline T-junction. Then, the RF power is divided equally, and the signals in different sub-circuits of the slotline T-junction have the same magnitude and $180^{\circ}$ out of phase. By adopting two pairs of symmetry L-shaped microstrip/slotline transition structures and a pair of SLRs, the proposed FPD can achieved a sharp bandpass response. In the end, two DM signals with the same magnitude and phase propagating along the slotline can be transmitted successfully into the output ports by U-type microstrip feedlines. The analysis of each part is provided in the following sections.

\section{B. Balanced Microstrip/Slotline Transition Structure}

The proposed FPD is fed by three balanced microstrip/slotline transition structures. Each structure comprises a U-type stepped-impedance microstrip feedline and a stepped-impedance slotline resonator. The steppedimpedance slotline resonator is etched on the bottom side of the substrate and is crossed at a right angle by a U-type microstrip line on the top side of the substrate. Meanwhile, the slotline resonator extends about a quarter of wavelength beyond the U-type microstrip feedline. The U-type microstrip line is coupled with the slotline by means of the magnetic field. In order to obtain a better impedance matching and

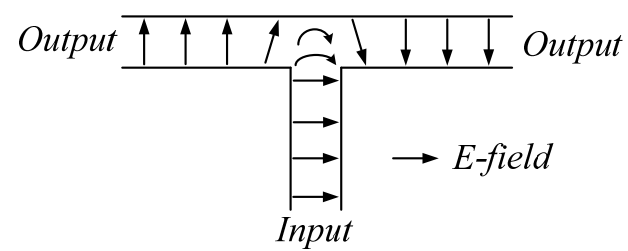

Fig. 8. The slotline T-junction with phase analysis.

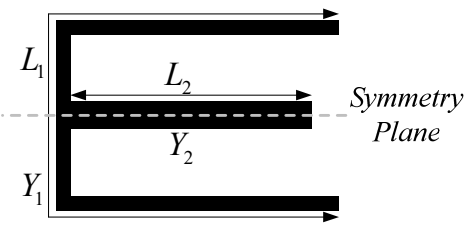

(a)

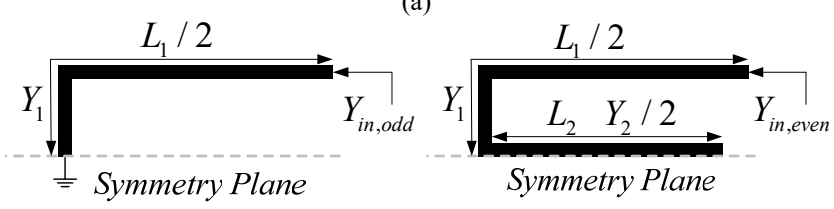

(b)

(c)

Fig. 9. (a) Configuration of the SLR. (b) Odd-mode equivalent circuit and (c) Even-mode equivalent circuit of SLR.

miniaturization, stepped-impedance microstrip line and slotline are adopted.

Because of the geometrical symmetry, the balanced circuit can be analyzed using the DM and CM method, as shown in Fig. 6. Furthermore, the cross-views of electrical field distribution at $T-T^{\prime}$ plane, which indicated in the Fig. 6, is shown in Fig. 7 and are herein used for demonstrating its working mechanism. When a DM excitation is applied, a virtual electrical wall can be obtained at plane $A-A^{\prime}$. A quasi transverse electromagnetic slotline mode generates a virtual electrical wall at the same plane, as illustrated in Fig. 7(a). Through strong magnetic coupling, the DM signals along the U-type microstrip line can be converted successfully into the slotline mode propagating along the slotline and be transferred to the next transition. Therefore, good DM transmission with a wideband response can be realized. The wideband transmission characteristic of slotline resonator have been analyzed in [9]. Under CM operation, a virtual magnetic wall at $T-T^{\prime}$ plane is formed, as shown in Fig. 7(b). Due to the magnetic wall perpendicular to the electric field of the slotline mode, which conflicts with the magnetic wall's boundary condition, the CM signals are blocked and a better CM suppression is achieved. In the end, balanced microstrip/slotline transition structures are employed to transformation between the slotline modes and the microstrip line modes. Therefore, the design process can be greatly simplified.

\section{Slotline T-Junction}

In this work, a uniform impedance slotline T-junction is introduced to achieve a ultra-wideband power division response [25]. The configuration with phase analysis of the proposed uniform impedance slotline T-junction is shown in Fig. 8. It can be seen that the two sub-circuits of the slotline $\mathrm{T}$-junction are opposite in phase, which means that an 


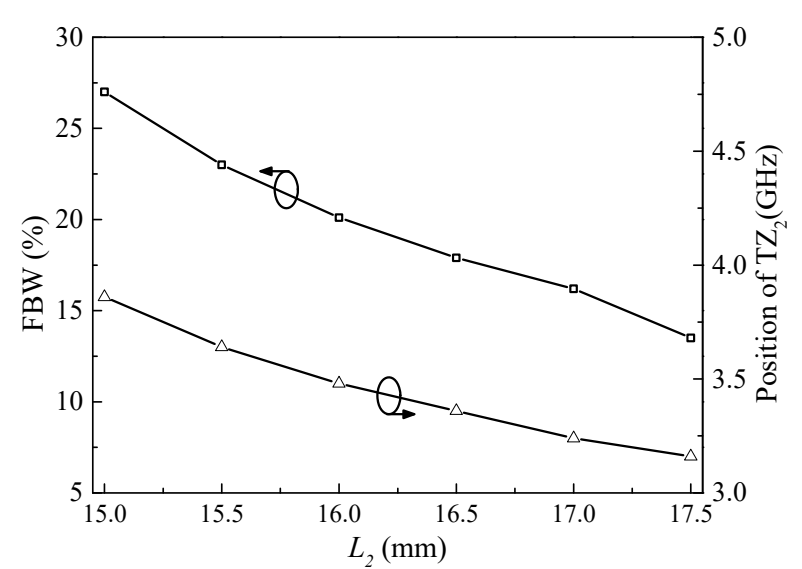

Fig. 10. The FBW change and the position change of $\mathrm{TZ}_{2}$ with different dimensions of $L_{2}$.

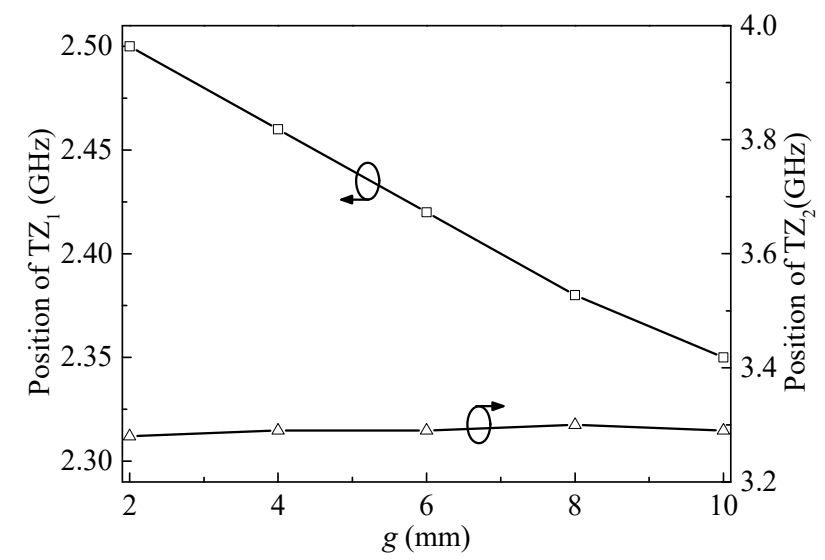

Fig. 11. The position change of $\mathrm{TZ}_{1}$ with different dimensions of $g$.

additional $180^{\circ}$ phase shift is introduced in the two signal paths. This can be explained qualitatively by considering the E-field distribution associated with the slotline T-junction transition, as shown in Fig. 8.

\section{Analysis of Bandpass Response}

The proposed FPD can achieve a good bandpass response by introducing a pair of symmetrical L-shaped microstrip/slotline transition structures and an SLR in each sub-circuit of slotline T-junction. The employed SLR consists of a half wavelength microstrip resonator and an open-stub loaded at the center, as shown in Fig. 9(a), where $Y_{1}, L_{1}, Y_{2}$ and $L_{2}$ denote the characteristic admittances and lengths of the microstrip line and open-stub, respectively. The open-stub is shunted at the middle of the microstrip line. Since the adopted resonator is symmetrical in structure, odd- and even-mode analysis can be adopted to characterize it.

For the odd-mode excitation, there is a voltage null along the middle of the resonator, which leads to an approximated equivalent circuit of Fig. 9(b). Therefore, the odd-mode resonant frequencies can be easily as:

$$
f_{\text {odd }}=\frac{(2 n-1) c}{2 L_{1} \sqrt{\varepsilon_{\text {eff }}}}
$$

where $n=1,2,3, \ldots, c$ is the speed of light in free space, and $\varepsilon_{e f f}$ denotes the effective dielectric constant of the substrate. It can

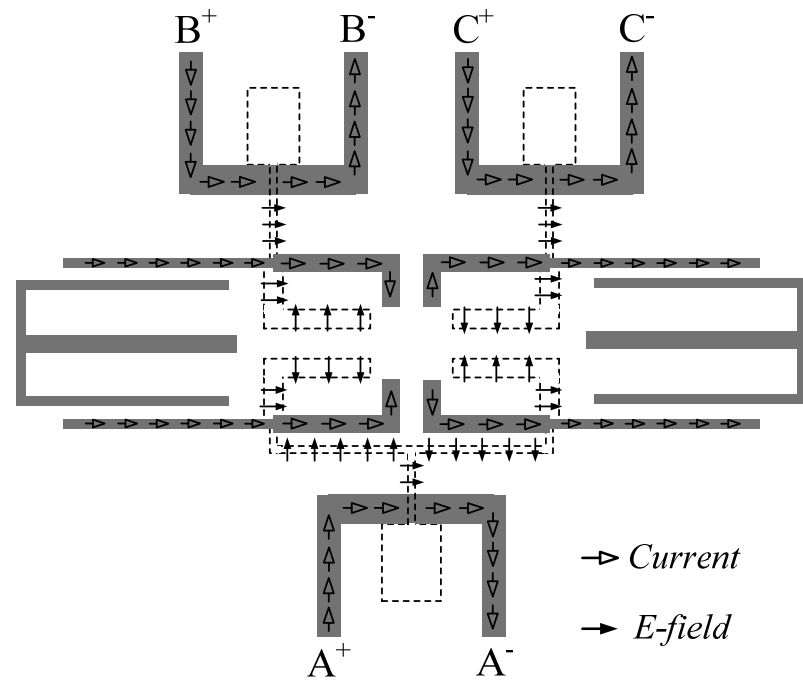

Fig. 12. The electric field distribution of the proposed FPD.

be observed that the odd-mode resonant frequencies are not affected by the open stub.

For the even-mode excitation, there is no current flow through the symmetrical plane. Thus we can bisect the circuit with open circuits at the middle to obtain an equivalent circuit of Fig. 9(c). The even-mode resonant frequencies for the case of $Y_{2}=2 Y_{1}$ can thus be attained as:

$$
f_{\text {even }}=\frac{n c}{\left(L_{1}+2 L_{2}\right) \sqrt{\varepsilon_{\text {eff }}}}
$$

According to the analysis above, the adopted SLR has two fundamental resonant frequencies. When $n=1$, the two fundamental resonant frequencies can be expressed as:

$$
\begin{aligned}
& f_{1}=f_{\text {odd }}=\frac{c}{2 L_{1} \sqrt{\varepsilon_{\text {eff }}}} \\
& f_{2}=f_{\text {even }}=\frac{c}{\left(L_{1}+2 L_{2}\right) \sqrt{\varepsilon_{\text {eff }}}}
\end{aligned}
$$

It can be noted that the bandwidth is easy to control by adjusting the interval between $f_{1}$ and $f_{2}$. And the center frequency $f_{c}$ can be roughly expressed as:

$$
f_{c}=\frac{f_{1}+f_{2}}{2}
$$

By properly adjusting the interval of two resonances of the DM response, a controllable bandwidth of the DM passband can be achieved. Therefore, both the center frequency and fractional bandwidth (FBW) of the proposed FPD are controllable and can be adjusted conveniently.

In addition, the adopted dual-mode SLR with the two adjacent L-shaped stepped-impedance microstrip lines can be viewed as an independent BPF unit, and two adjacent L-shaped microstrip lines can be simply viewed as a source and a load of the BPF. By taking advantages of the cross coupling between the slotlines, which locate below the L-shaped microstrip lines, illustrated in Fig. 2, one TZ can be engendered at the lower frequency outside the passband to obtain a sharp roll-off skirt. The second $\mathrm{TZ}$ is created because of the intrinsic characteristics of the embedded SLR, and the position of the $\mathrm{TZ}_{2}$ has a direct 
correlation with the length of the open-stub. Therefore, the selectivity of the bandpass response can be greatly improved by introducing the $\mathrm{TZ}_{1}$ and $\mathrm{TZ}_{2}$, which located at each side of the passband respectively.

With the above analysis, the position of $\mathrm{TZ}_{2}$ and the FBW of the passband can be adjusted by changing the length of the open-stub conveniently. When the length $L_{l}$ is fixed at $42 \mathrm{~mm}$ (corresponding to $\lambda_{g} / 2$ at the fundamental odd-mode resonant frequency $f_{1}$ ), by changing the open-stub length $L_{2}$, the fundamental even-mode resonant frequency $f_{2}$ and $\mathrm{TZ}_{2}$ can be shifted accordingly, whereas $f_{1}$ and $\mathrm{TZ}_{1}$ is maintained unchanged. Therefore, the FBW and $\mathrm{TZ}_{2}$ can be controlled by adjusting the lengths of open-stub, which is simulated as shown in Fig. 10. Meanwhile, the position of $\mathrm{TZ}_{1}$ can be adjusted by changing the gap $(g)$ of slotlines, which is indicated in Fig. 2, and the position of $\mathrm{TZ}_{1}$ remains approximately unchanged, which is simulated as shown in Fig. 11.

\section{The Proposed FPD With High Isolation PERFORMANCE}

Based on the design method presented above, a balanced power divider with a DM bandpass response can be realized. However, the proposed FPD suffers from poor DM isolation performance between the output ports due to the introduction of the slotline T-junction structure. To improve the isolation performance, a novel isolation circuit is introduced.

\section{A. Overall Analysis of Phase Change}

One challenge for the proposed FPD is that the isolation cannot be simply improved by using a resistor when it is connected directly across the two branches or output ports as in the modified Wilkinson structures or other configurations. This is because of the $180^{\circ}$ phase difference of the L-shaped microstrip lines. To address this issue, it is necessary to make a detailed analysis of phase change in different branches of the proposed FPD.

The electric field and current distributions in the slotlines and microstrip lines (not including the SLR) are shown in Fig. 12, respectively, which enables us to have a clear understanding of the in-phase and out-of-phase coupling behavior of the proposed FPD. It can be clearly seen that the signals at the two balanced output ports are in-phase. On the other hand, the signals in the adjacent L-shaped microstrip lines, which can be used to connect resistors easily, have the same magnitude but are $180^{\circ}$ out of phase. Therefore, the resistor cannot provide any effect if it's connected directly across the adjacent L-shaped microstrip lines.

\section{B. Improvement of Isolation Performance}

Based on the above phase analysis, a novel isolation structure based on slotline loaded with resistor is proposed. The proposed isolation structure consists of a uniform impedance slotline and a resistor connected across the midpoint of the slotline. Fig. 13 shows the configuration of the proposed in-phase FPD with the proposed isolation structure to improved isolation performance. Based on the previous analysis on U-type balanced input/output ports, only DM equivalent circuit

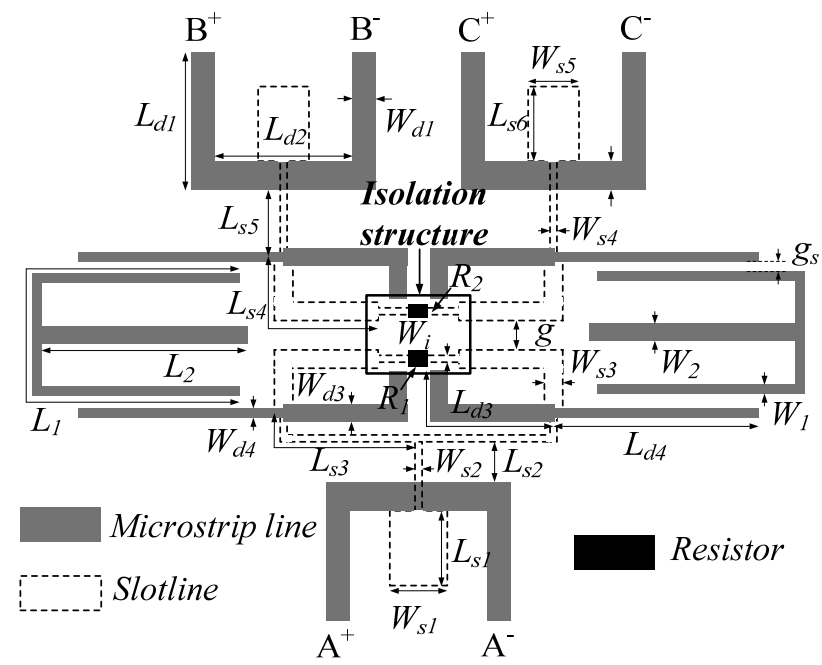

Fig. 13. Configuration of the proposed FPD with the proper structure to improve the isolation performance.

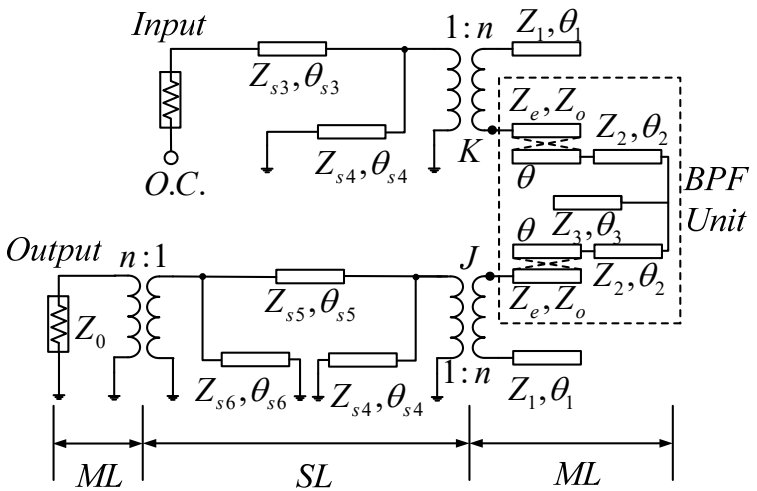

(a)

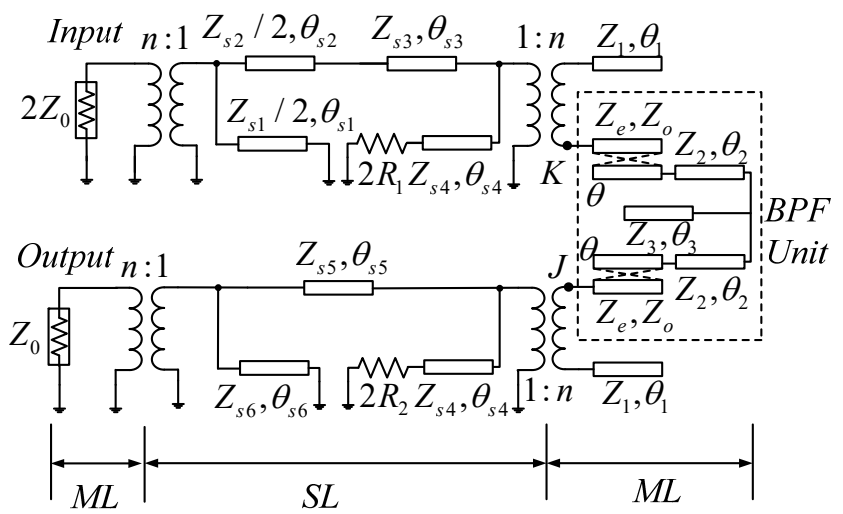

(b)

Fig. 14. (a) Even-mode equivalent impedance-matching circuit and (b) Odd-mode equivalent impedance-matching circuit of the proposed FPD with improved isolation performance under DM operation. (ML = Microstrip Line; $\mathrm{SL}=$ Slotline $)$

needs to be considered in the following design process. In this case, the proposed FPD can be simply viewed as a three-port network, as shown in Fig. 4. Apparently, owing to the symmetrical structure, the odd- and even-mode method can be employed to analyze the presented DM equivalent circuit in consideration of the phase change of the slotline T-junction.

Fig. 14(a) shows the even-mode equivalent impedance matching circuit of the proposed FPD with high isolation 
performance. Under DM operation, two signals with the same magnitude and $180^{\circ}$ out of phase are applied to each output port. In this case, the signals propagating along the slotline on each side of resistors are in-phase. Hence, the isolation resistors loaded on the slotline can be omitted. Meanwhile, since the signals in the two sub-circuits of the slotline T-junction have the same magnitude and phase, no current flows through the input slotline of slotline T-junction.

The two-port network between nodes $K$ and $J$ in Fig. 14 can be calculated independently. The $\theta$ represents the electric lengths of the even- and odd-mode, which can be viewed as equal to simplify the next calculation process. Therefore, the ABCD-matrix of the two-port network between nodes $K$ and $J$ can be calculated as:

$$
\begin{aligned}
& {\left[\begin{array}{ll}
A & B \\
C & D
\end{array}\right]=\left[\begin{array}{cc}
\frac{c \cos \theta}{d} & j \frac{d^{2}-c^{2} \cos ^{2} \theta}{2 d \sin \theta} \\
j \frac{2 \sin \theta}{d} & \frac{c \cos \theta}{d}
\end{array}\right]} \\
& \times\left[\begin{array}{cc}
\cos \theta_{2} & j Z_{2} \sin \theta_{2} \\
j \frac{1}{Z_{2}} \sin \theta_{1} & \cos \theta_{2}
\end{array}\right]\left[\begin{array}{cc}
1 & 0 \\
j \frac{1}{Z_{3}} \tan \theta_{3} & 1
\end{array}\right] \\
& \times\left[\begin{array}{cc}
\cos \theta_{2} & j Z_{2} \sin \theta_{2} \\
j \frac{1}{Z_{2}} \sin \theta_{2} & \cos \theta_{2}
\end{array}\right]\left[\begin{array}{cc}
\frac{c \cos \theta}{d} & j \frac{d^{2}-c^{2} \cos ^{2} \theta}{2 d \sin \theta} \\
j \frac{2 \sin \theta}{d} & \frac{c \cos \theta}{d}
\end{array}\right]
\end{aligned}
$$

and

$$
c=Z_{e}+Z_{o} \quad d=Z_{e}-Z_{o}
$$

In order to further simplify the design process and to select appropriate isolation resistors, the input and output impedances of the two-port network between nodes $K$ and $J$ can be set as $Z_{F}$ in consideration of its symmetrical structure.

Furthermore, when $\theta_{1}=\theta_{s 4}=\theta_{s 6}=\pi / 2$, the equivalent impedance of the part before the node $K$ can be calculated as:

$$
Z_{i}^{e}=n^{2} \frac{Z_{s 3}}{j \tan \theta_{s 3}}
$$

and equivalent impedance of the part after the node $J$ can be calculated as:

$$
Z_{t}^{e}=n^{2} \frac{Z_{0}+n^{2} j Z_{s 5} \tan \theta_{s 5}}{j \frac{Z_{0}}{Z_{s 5}} \tan \theta_{s 5}+n^{2}}
$$

Therefore, in order to obtain good input/output impedance matching, the following relationship has to be satisfied:

$$
\frac{Z_{s 3}}{j \tan \theta_{s 3}}=Z_{F}=\frac{Z_{0}+n^{2} j Z_{s 5} \tan \theta_{s 5}}{j \frac{Z_{0}}{Z_{s 5}} \tan \theta_{s 5}+n^{2}}
$$

In this case, if $Z_{s 3}=Z_{s 5}=Z_{0}$, the following expression can be further simplified as:

$$
j \tan \left(\theta_{s 3}-\theta_{s 5}\right)=n^{2}
$$

which provides a guideline in the selection of the $\theta_{s 3}$ and $\theta_{s 5}$.

Fig. 14(b) shows the odd-mode equivalent impedance matching circuit correspondingly. In this case, two signals with the same magnitude and phase are applied to each output port.

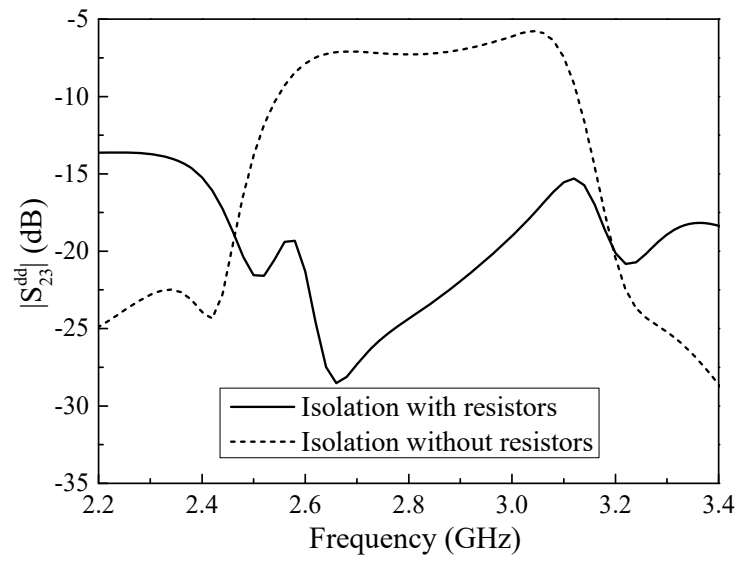

Fig. 15. The comparison of DM isolation of the proposed FPD with resistors and without resistors.

However, the signals propagating along the slotline on each side of isolation resistors are $180^{\circ}$ out-of-phase. Therefore, the isolation resistors can improve the isolation between the two output ports. In the bisected circuit, the isolation resistor $R$ is doubled $(2 R)$. When the input port impedance $\left(2 Z_{0}\right)$ is equal to the output port impedance, the impedance matching circuit can be achieved.

Therefore, when $\theta_{1}=\theta_{s 1}=\theta_{s 4}=\theta_{s 6}=\pi / 2$, the equivalent impedances before the node $K$ and after the node $J$ can be calculated respectively as:

$$
Z_{i}^{o}=n^{2} \frac{1}{\frac{1}{Z_{t}^{o}}+\frac{2 R_{1}}{Z_{s 4}^{2}}}
$$

where

$$
Z_{t}^{o}=\frac{\frac{4 Z_{0}+j n^{2} Z_{s 2} \tan \theta_{s 2}}{j \frac{8 Z_{0}}{Z_{s 2}} \tan \theta_{s 2}+2 n^{2}}+j Z_{s 3} \tan \theta_{s 3}}{j \frac{1}{Z_{s 3}} \tan \theta_{s 3} \frac{4 Z_{0}+j n^{2} Z_{s 2} \tan \theta_{s 2}}{j \frac{8 Z_{0}}{Z_{s 2}} \tan \theta_{s 2}+2 n^{2}}+1}
$$

and

$$
Z_{l}^{o}=n^{2} \frac{1}{\frac{j Z_{0} \tan \theta_{s 5}+Z_{s 5}}{Z_{0} Z_{s 5}+j n^{2} Z_{s 5}^{2} \tan \theta_{s 5}}+\frac{2 R_{2}}{Z_{s 4}^{2}}}
$$

To obtain a good input/output impedance match, the following relationship have to be satisfied:

$$
\frac{1}{Z_{t}^{o}}+\frac{2 R_{1}}{Z_{s 4}^{2}}=\frac{n^{2}}{Z_{F}}=\frac{j Z_{0} \tan \theta_{s 5}+Z_{s 5}}{Z_{0} Z_{s 5}+j n^{2} Z_{s 5}^{2} \tan \theta_{s 5}}+\frac{2 R_{2}}{Z_{s 4}^{2}}
$$

which provides a guideline in the selection of the parameters. Furthermore, if $\theta_{s 2} \approx 0, Z_{s 2}=Z_{s 3}=Z_{s 5}=Z_{0}$, and $Z_{F}=Z_{s 4}=2 Z_{0}$, the above equation can be simplifies to the following:

$$
\frac{R_{1}}{2 Z_{0}}=\frac{n^{2}}{2}-\frac{j 2 \tan \theta_{s 3}+n^{2}}{2+j n^{2} \tan \theta_{s 3}}
$$

and

$$
\frac{R_{2}}{2 Z_{0}}=\frac{n^{2}}{2}-\frac{j \tan \theta_{s 5}+1}{1+j n^{2} \tan \theta_{s 5}}
$$




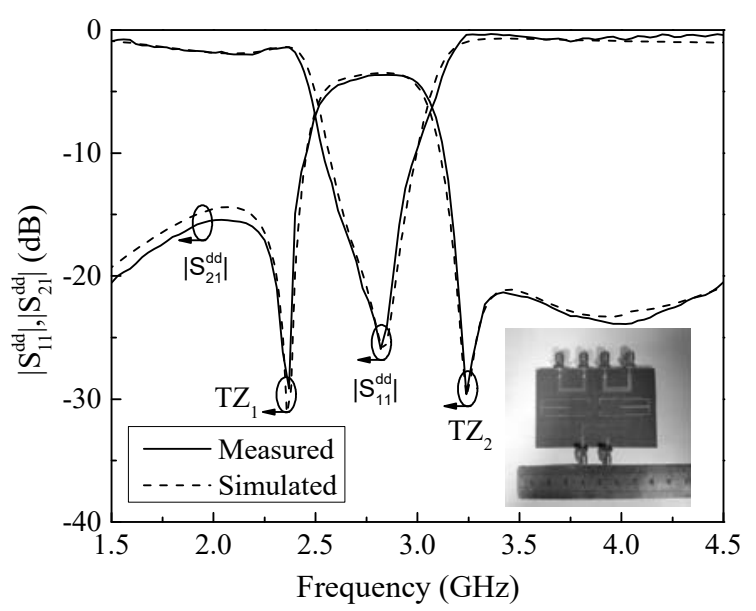

(a)

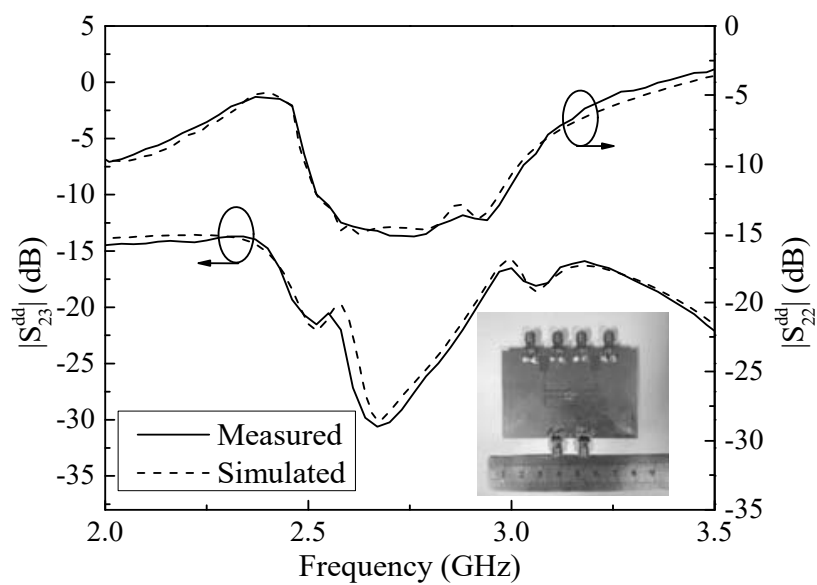

(b)

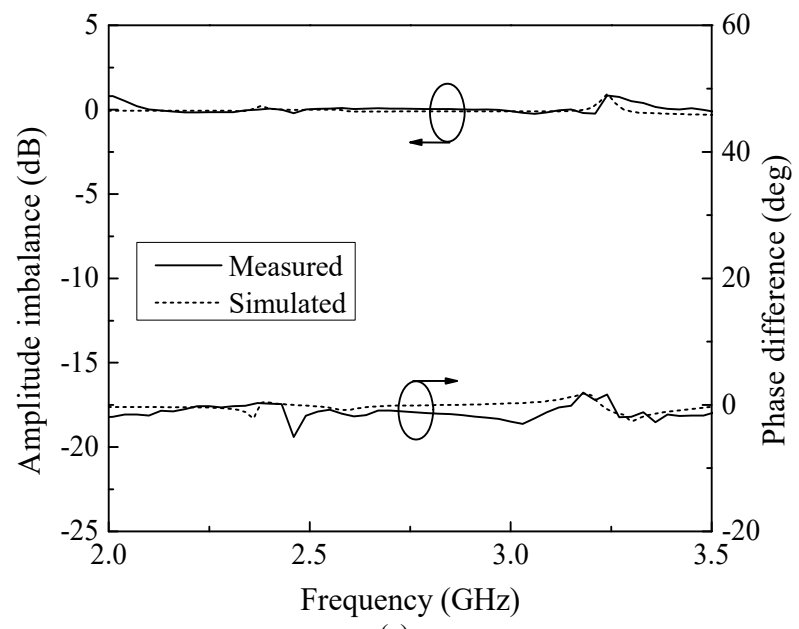

(c)

Fig. 16. Comparisons of the simulated and measured results. (a) DM return loss and insert loss, (b) DM isolation and output impedance matching, and (c) DM amplitude imbalance and phase difference between the two output ports. (Narrow passband)

Similar to [23], resistors can be selected as:

$$
R_{1}=R_{2}=2 Z_{0}
$$

Then, good DM isolation between the two output ports can be achieved. The improved isolation performance can be

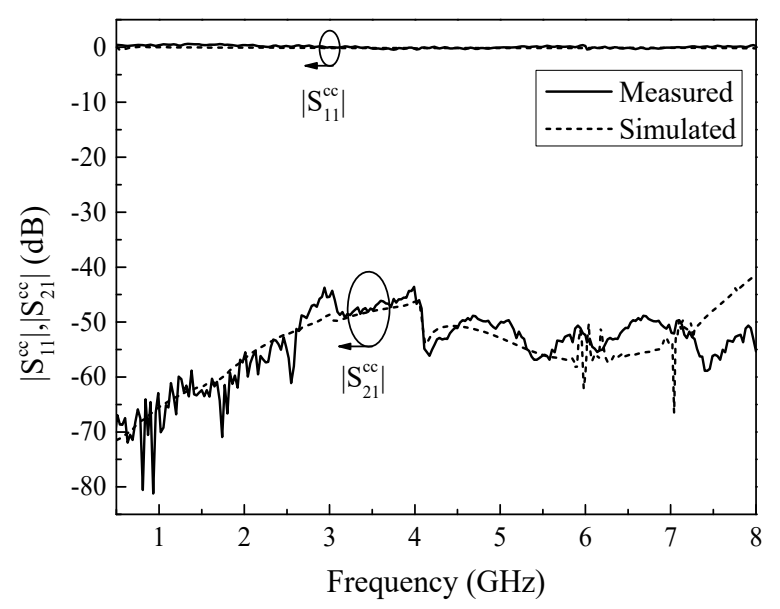

Fig. 17. Comparisons of the simulated and measured results of $\mathrm{CM}$ responses. (Narrow Passband)

clearly seen in Fig. 15.

Finally, by adopting the befitting design and optimizing method, a balanced-to-balanced in-phase FPD with high selectivity and isolation performance can be realized.

\section{Design Procedure}

Based on the above analysis and discussions, a simple design procedure of the proposed balanced-to-balanced FPD can be summarized as follows.

Step 1) For the desired center frequency and filter bandwidth, the physical widths and lengths of every line sections of the SLR can be selected appropriately according to (5), (6), and (7).

Step 2) According to (12) and (17), realizable electrical lengths and characteristic impedances of microstrip lines and slotlines can be chosen.

Step 3) According to (18) and (19), realizable $R_{1}$ and $R_{2}$ can be found.

Step 4) Further optimize all values to realize better DM and $\mathrm{CM}$ responses and isolation performance of the proposed FPD. Full-wave electromagnetic simulation and dimension optimization in the commercial software of HFSS can be conducted.

\section{Simulated And Measured Results Of The FABRICATED BALANCED FPDS With High SELECTIVITY AND ISOLATION PERFORMANCE}

A balanced-to-balanced two-way in-phase equal FPD with high selectivity and isolation performance has been designed according to the above design method. A narrowband FPD and a wideband one have been fabricated and measured to verify the feasibility of the proposed design method under different bandwidth requirements. Meanwhile, by properly adjusting the size of the SLR, the proposed FPDs with different center frequencies and bandwidths can be easily achieved. The substrate used is F4BM-2 with a thickness of $0.8 \mathrm{~mm}$ and a dielectric constant of 2.2. The S-parameters of the proposed and fabricated FPDs were measured with an Agilent VNA (vector network analyzer) N5230. 


\section{A. The Fabricated FPD With A Narrow Passband}

The effective dimension of the fabricated balanced FPD with a narrow passband is only $63.3 \mathrm{~mm} \times 47.3 \mathrm{~mm}\left(0.60 \lambda_{g} \times 0.45 \lambda_{g}\right.$, where $\lambda_{g}$ is the guide wavelength at $\left.2.85 \mathrm{GHz}\right)$, which all the dimensions are selected as follows: $W_{d 1}=2.4 \mathrm{~mm}, W_{d 2}=3.0$ $\mathrm{mm}, W_{d 3}=1.2 \mathrm{~mm}, W_{d 4}=0.5 \mathrm{~mm}, W_{1}=0.5 \mathrm{~mm}, W_{2}=1.2 \mathrm{~mm}$, $W_{s 1}=6.0 \mathrm{~mm}, W_{s 2}=0.2 \mathrm{~mm}, W_{s 3}=1.0 \mathrm{~mm}, W_{s 4}=0.2 \mathrm{~mm}, W_{s 5}$ $=5.0 \mathrm{~mm}, W_{i}=0.2 \mathrm{~mm}, L_{d 1}=15 \mathrm{~mm}, L_{d 2}=14.4 \mathrm{~mm}, L_{d 3}=12.4$ $\mathrm{mm}, L_{d 4}=16.0 \mathrm{~mm}, L_{1}=42.0 \mathrm{~mm}, L_{2}=17.0 \mathrm{~mm}, L_{s 1}=8.0 \mathrm{~mm}$, $L_{s 2}=3.0 \mathrm{~mm}, L_{s 3}=12.2 \mathrm{~mm}, L_{s 4}=13.5 \mathrm{~mm}, L_{s 5}=5.0 \mathrm{~mm}, L_{s 6}=$ $7.5 \mathrm{~mm}, g_{s}=0.3 \mathrm{~mm}, g=2 \mathrm{~mm}$. Two surface-mount resistors $(0402,100 \Omega)$ from muRata Corporation are employed.

The simulated and measured S-parameters are presented in Fig. 16 and Fig. 17. It can be seen that the measured results agree well with the simulated ones over the entire operation frequency range. The measured impedance bandwidth (10-dB bandwidth) is from $2.54 \mathrm{GHz}$ to $2.98 \mathrm{GHz}$ (FBW $=15.9 \%$, which is calculated at centre frequency of $2.76 \mathrm{GHz}$ ). The measured DM insertion loss (the $3 \mathrm{~dB}$ power division intrinsic loss is not included) is less than $0.6 \mathrm{~dB}$ and the measured maximum DM reflection loss is better than $25 \mathrm{~dB}$ over the DM passband, as shown in Fig. 16(a). Meanwhile, two TZs at 2.37 $\mathrm{GHz}$ and $3.24 \mathrm{GHz}$ are located at the both sides of the passband, respectively. As shown in Fig. 16(b), the measured DM isolation is better than $18 \mathrm{~dB}$ around the $\mathrm{DM}$ passband, and the measured maximum isolation is better than $32 \mathrm{~dB}$ for DM signals. Meanwhile, a good impedance matching of output port is achieved $(>13.4 \mathrm{~dB})$. The simulated and measured amplitude imbalance and phase difference between the balanced output ports are better than $0.3 \mathrm{~dB}$ and $3^{\circ}$ within the operating band, respectively, as shown in Fig.16(c).

For CM signals, a broad bandwidth $(\mathrm{FBW}=271.7 \%)$ and a high suppression (greater than $45 \mathrm{~dB}$ ) are achieved both in the simulation and measurement, as shown in Fig. 17.

\section{B. The Fabricated FPD With A Wide Passband}

The fabricated balanced wideband FPD has the same overall effective dimension as the narrowband one. All the dimensions of the proposed balanced FPD with a wide passband are selected as follows: $W_{d 1}=2.4 \mathrm{~mm}, W_{d 2}=3.0 \mathrm{~mm}, W_{d 3}=1.2 \mathrm{~mm}$, $W_{d 4}=0.5 \mathrm{~mm}, W_{1}=0.5 \mathrm{~mm}, W_{2}=1.2 \mathrm{~mm}, W_{s 1}=6.0 \mathrm{~mm}, W_{s 2}=$ $0.2 \mathrm{~mm}, W_{s 3}=1.0 \mathrm{~mm}, W_{s 4}=0.2 \mathrm{~mm}, W_{s 5}=5.0 \mathrm{~mm}, W_{i}=0.2$ $\mathrm{mm}, L_{d 1}=15 \mathrm{~mm}, L_{d 2}=14.4 \mathrm{~mm}, L_{d 3}=12.4 \mathrm{~mm}, L_{d 4}=16.0$ $\mathrm{mm}, L_{1}=42.0 \mathrm{~mm}, L_{2}=15.5 \mathrm{~mm}, L_{s 1}=8.0 \mathrm{~mm}, L_{s 2}=3.0 \mathrm{~mm}$, $L_{s 3}=12.2 \mathrm{~mm}, L_{s 4}=13.5 \mathrm{~mm}, L_{s 5}=5.0 \mathrm{~mm}, L_{s 6}=7.5 \mathrm{~mm}, g_{s}=$ $0.3 \mathrm{~mm}, g=2 \mathrm{~mm}$. Two surface-mount resistors $(0402,100 \Omega)$ from muRata Corporation are employed.

The comparison of simulated and measured DM responses is presented in Fig. 18. It can be seen that good agreement of the measured and simulated results can be achieved around the passband. A wide DM operating band (10-dB bandwidth) is obtained covering from $2.65 \mathrm{GHz}$ to $3.35 \mathrm{GHz}(\mathrm{FBW}=23.3 \%$ which is calculated at centre frequency of $3.0 \mathrm{GHz})$. The measured DM insertion loss (the $3 \mathrm{~dB}$ power division intrinsic loss is not included) is less than $0.8 \mathrm{~dB}$ and the measured maximum DM reflection loss is better than $15 \mathrm{~dB}$ within the DM passband, as shown in Fig. 18(a). Meanwhile, two TZs at

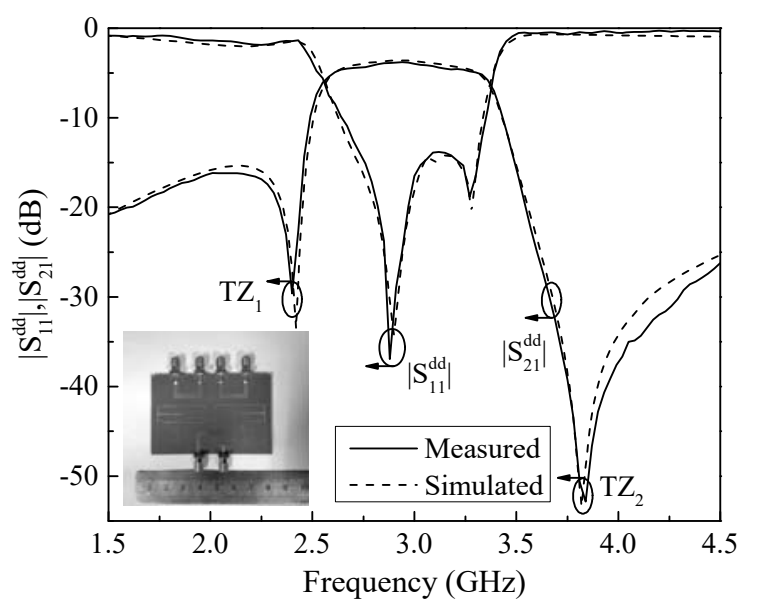

(a)

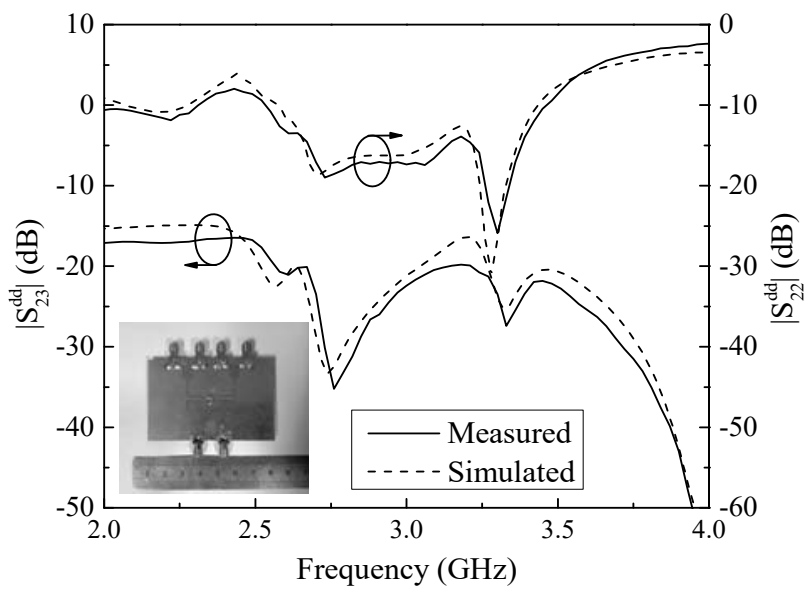

(b)

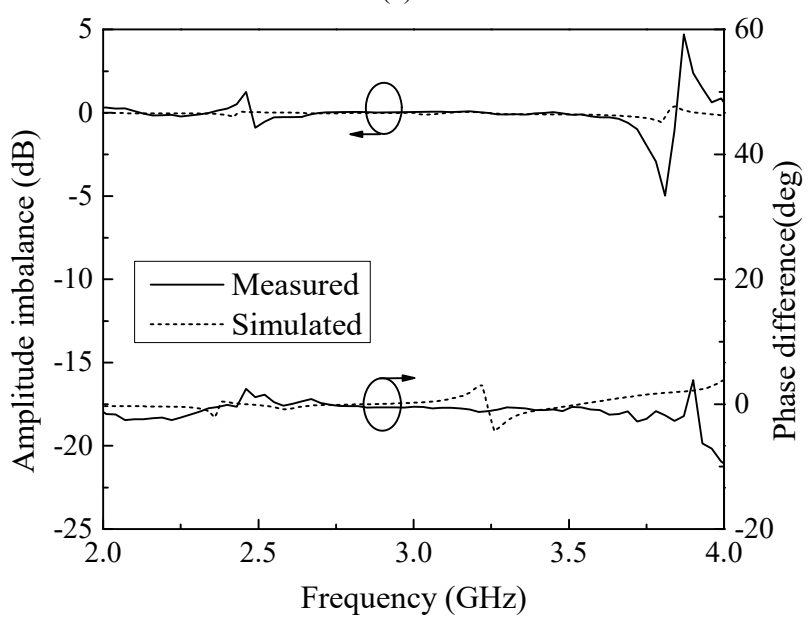

(c)

Fig. 18. Comparisons of the simulated and measured results. (a) DM return loss and insert loss, (b) DM isolation and output impedance matching, and (c) DM amplitude imbalance and phase difference between the two output ports. (Wide passband)

$2.40 \mathrm{GHz}$ and $3.84 \mathrm{GHz}$ can be achieved, which are located at the both sides of the passband, respectively. In the design of the balanced FPD with a wideband DM response, some acceptable in-band ripples appear. This can be caused by the distance between the two poles of the DM passband. As shown in Fig. $18(\mathrm{~b})$, the measured DM isolation is better than $20 \mathrm{~dB}$ around the DM passband, and the measured maximum isolation is 


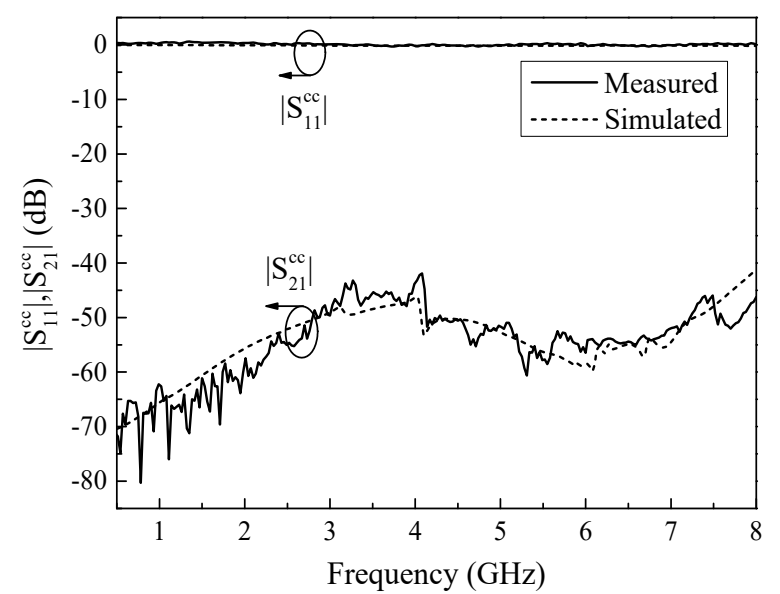

Fig. 19. Comparisons of the simulated and measured results of $\mathrm{CM}$ responses. (Wide passband)

TABLE I

COMPARISON WITH REPORTED BALANCED-TO-BALANCED FPD

\begin{tabular}{|c|c|c|c|c|c|c|}
\hline & $\begin{array}{c}f_{0}(\mathrm{GHz}) / \\
3 \mathrm{~dB} \\
\text { FBW }(\%)\end{array}$ & $\begin{array}{c}\mathrm{IL} \\
(\mathrm{dB})\end{array}$ & $\begin{array}{c}\text { No. of } \\
\text { TZs }\end{array}$ & $\begin{array}{l}\text { Iso } \\
\text { (dB) }\end{array}$ & $\begin{array}{c}\text { CMS } \\
(\mathrm{dB}) / \mathrm{CM} \\
\text { FBW }(\%)\end{array}$ & $\begin{array}{l}\text { Size } \\
\left(\lambda_{\mathrm{g}}{ }^{2}\right)\end{array}$ \\
\hline $\begin{array}{c}\text { Design I } \\
\text { in [19] }\end{array}$ & $2.02 / 8.4$ & 0.73 & 0 & 19 & $20 / 70$ & $\begin{array}{c}0.97 \times 0 \\
87\end{array}$ \\
\hline $\begin{array}{c}\text { Design II } \\
\text { in [19] }\end{array}$ & $2.01 / 7.7$ & 0.86 & 1 & 27.4 & $20 / 60$ & $\begin{array}{c}0.67 \times 0 \\
63\end{array}$ \\
\hline [20] & $2.39 / 7$ & 2.21 & 1 & 20 & $60 / 209$ & $\begin{array}{c}0.46 \times 0 \\
40\end{array}$ \\
\hline [21] & $\begin{array}{c}2.39 / 5.5 \\
\left(\left|S_{11}^{d d}\right|\right. \\
<10 \mathrm{~dB})\end{array}$ & 3.6 & 0 & 9.1 & $19 / 125$ & $\begin{array}{c}0.29 \times 0 \\
28\end{array}$ \\
\hline [22] & $\begin{array}{c}2.45 / 6.1 \\
\left(\left|S_{11}^{d d}\right|\right. \\
<10 \mathrm{~dB})\end{array}$ & 1.0 & 0 & 40 & $20 / 205$ & $\begin{array}{c}\text { Unkno } \\
\text { wn }\end{array}$ \\
\hline Design I & $\begin{array}{c}2.76 / 15.9 \\
\left(\left|S_{11}^{d d}\right|\right. \\
<10 \mathrm{~dB})\end{array}$ & 0.6 & 2 & 18 & $45 / 271.7$ & $\begin{array}{c}0.60 \times 0 \\
45\end{array}$ \\
\hline Design II & $\begin{array}{c}3.0 / 23.3 \\
\left(\left|S_{11}^{d d}\right|\right. \\
<10 \mathrm{~dB})\end{array}$ & 0.8 & 2 & 20 & $45 / 250$ & $\begin{array}{c}0.60 \times 0 \\
45\end{array}$ \\
\hline
\end{tabular}

Iso = Isolation; CMS = Common-mode Suppression

better than $35 \mathrm{~dB}$. Meanwhile, a good impedance matching of output port is achieved $(>12.5 \mathrm{~dB})$. The simulated and measured amplitude imbalance and phase difference between the balanced output ports, which presented in Fig. 18(c), are less than $0.3 \mathrm{~dB}$ and $3.5^{\circ}$ within the operating band, respectively. Fig. 19 shows the simulated and measured CM responses of the wideband balanced FPD. It can be seen that a broad bandwidth (FBW $=250 \%$ ) and a high CM suppression (more than $45 \mathrm{~dB}$ ) are achieved both in the simulation and measurement. It can be noted that the $\mathrm{CM}$ responses is independent from the DM responses.

A comparison with some previously reported balanced-to- balanced FPDs is available in Table I. It is seen that the proposed FPD has overall superior performance in terms of IL, selectivity and CM suppression. The better performance and simpler structure make it attractive and competitive for balanced wireless communications systems.

\section{CONCLUSION}

In this paper, a balanced-to-balanced two-way in-phase filtering power divider with superior performances has been designed and analyzed. Meanwhile, two proposed balanced FPDs with different bandwidth values have been fabricated and measured. The simulated results and the measured results agree very well. The simulated and measured results indicate that the proposed balanced FPDs have advantages of compact size, high CM suppression, high selectivity, better DM in-band isolation, and good output impedance-matching. Moreover, the proposed FPD has a great flexibility in filtering design, which makes it suitable for various balanced systems.

\section{REFERENCES}

[1] J. Shi and Q. Xue, "Balanced bandpass filters using center-loaded half-wavelength resonators," IEEE Trans. Microw. Theory Techn., vol. 58, no. 4, pp. 970-977, Apr. 2010.

[2] J. Shi and Q. Xue, "Dual-Band and wide-stopband single-band balanced bandpass filters with high selectivity and common-mode suppression," IEEE Trans. Microw. Theory Techn., vol. 58, no. 8, pp. 2204-2212, Aug. 2010.

[3] J. Qiang, J. Shi, Q. H. Cao, and Z. D. Shen, "Compact differential wideband bandpass filters based on half-wavelength lines," IEEE Microw. Wireless Compon. Lett., vol. 27, no. 10, pp. 906-908, Oct. 2017.

[4] J.-X. Chen, M.-Z. Du, Y.-L. Li, Y.-J. Yang, and J. Shi, "Independently tunable/controllable differential dual-Band bandpass filters using element-loaded stepped-impedance resonators," IEEE Trans. Compon. Packag. Manuf. Technol., pp. 1-8, Nov, 2017.

[5] Y.-J. Lu, S. Yuan, and P. Hsu, "A differential-mode wideband bandpass filter with enhanced common-mode suppression using slotline resonator," IEEE Microw. Wireless Compon. Lett., vol. 22, no. 10, pp. 503-505, Oct. 2012.

[6] W. J. Feng, C. Y. Zhao, W. Q. Che, and Q. Xue, "A balanced-to-balanced network with unequal power division and wideband common mode suppression," IEEE Microw. Wireless Compon. Lett., vol. 26, no. 4, pp. 237-239, Apr. 2016.

[7] B. Xia, L.-S. Wu, S.-W. Ren, and J.-F. Mao, "A balanced-to-balanced power divider with arbitrary power division," IEEE Trans. Microw. Theory Techn., vol. 61, no. 8, pp. 2831-2840, Aug. 2015.

[8] B.-F. Su and T.-G. Ma, "Miniaturized common-mode filter using coupled synthesized lines and mushroom resonators for high-speed differential signals," IEEE Microw. Wireless Compon. Lett., vol. 25, no. 2, pp. 112-114, Feb. 2015.

[9] X. Guo, L. Zhu, J. P. Wang, and W. Wu "Wideband microstrip-tomicrostrip vertical transitions via multiresonant modes in a slotline resonator," IEEE Trans. Microw. Theory Techn., vol. 63, no. 6, pp. 1902-1909, Jun. 2015.

[10] L. Yang, L. Zhu, and W.-W Choi, and K.-W. Tam, "Analysis and design of wideband microstrip-to-microstrip equal ripple vertical transitions and their application to bandpass filters," IEEE Trans. Microw. Theory Techn., vol. 65, no. 8, pp. 2866-2877, Aug. 2017.

[11] J. Shi, C. Shao, J.-X. Chen, Q.-Y. Lu, Y. J. Peng, and Z.-H. Bao, "Compact low-loss wideband differential bandpass filter with high common-mode suppression," IEEE Microw. Wireless Compon. Lett., vol. 23, no. 9, pp. 480-482, Sep. 2013.

[12] J. Shi, J. Wang, K. Xu, J.-X. Chen, and W. Liu, "A balanced-to-balanced power divider with wide bandwidth," IEEE Microw. Wireless Compon. Lett., vol. 25, no. 9, pp. 573-575, Sep. 2015.

[13] W. J. Feng, C. Y. Zhao, W. Q. Che, and Q. Xue, "Wideband balanced network with high isolation using double-sided parallel-strip Line," IEEE Trans. Microw. Theory Techn., vol. 63, no. 12, pp. 4013-4018, Dec. 2015. 
[14] J. Shi and K. Xu, "Compact differential power divider with enhanced bandwidth and in-phase or out-of-phase output ports," Electron. Lett., vol. 50, no. 17, pp. 1209-1211, Aug. 2014.

[15] J. Shi, K. Xu, W. Zhang, J.-X. Chen, and G. H. Zhai, "An approach to 1-to- $2^{n}$ way microstrip balanced power divider," IEEE Trans. Microw. Theory Techn., vol. 64, no. 12, pp. 4222-4231, Dec. 2016.

[16] X. Gao, W. J. Feng, W. Q. Che, and Q. Xue, "Wideband balanced-to-unbalanced filtering power dividers based on coupled lines," IEEE Trans. Microw. Theory Techn., vol. 65, no. 1, pp. 86-95, Jan. 2017.

[17] K. Xu, J. Shi, L. L. Lin, and J.-X. Chen, "A balanced-to-unbalanced microstrip power divider with filtering function," IEEE Trans. Microw. Theory Techn., vol. 63, no. 8, pp. 2561-2569, Aug. 2015.

[18] W. J. Feng, M. L. Hong, and W. Q. Che "Dual-band balanced-to-unbalanced filtering power divider by coupled ring resonators," Electron. Lett., vol. 52, no. 22, pp. 1862-1864, Oct. 2016.

[19] L.-S. Wu, Y.-X. Guo, and J.-F. Mao, "Balanced-to-balanced Gysel power divider with bandpass filtering response," IEEE Trans. Microw. Theory Techn., vol. 61, no. 12, pp. 4052-4062, Dec. 2013.

[20] M. Luo, X. Xu, X.-H. Tang, and Y.-H. Zhang, "A compact balanced-to-balanced filtering Gysel power divider using $\lambda_{g} / 2$ resonators and short-stub-loaded resonator," IEEE Microw. Wireless Compon. Lett., vol. 27, no. 7, pp. 645-647, Jul. 2017.

[21] C.-L. Huang and Y.-H. Pang, "Filtering Power Divider for Differential Input and Output Signals," Proc. Asia-Pacific Microw. Conf. (APMC), pp. 369-371, Nov. 2013.

[22] W. J. Feng, X. K. Ma, W. Q. Che, and Y. L. Wu, "Narrow-band balanced filtering network using coupled lines loaded with stubs," Electron. Lett., vol. 54, no.6, pp. 366-368, Mar. 2018.

[23] K. J. Song, Y. X. Mo, Q. Xue, and Y. Fan, "Wideband four-way out-of-phase slotline power dividers," IEEE Trans. Ind. Electron., vol. 61 , no. 7, pp. 3598-3606, Jul. 2014.

[24] K. C. Gupta, R. Garg, I. Bahl, and P. Bhartia, "Microstrip Lines and Slotlines", 2nd ed. Norwood, MA, USA: Artech House, 1996.

[25] F. Wei, X. B. Zhao, X. Y. Wang, B. Li, and X. W. Shi, "A balanced UWB power divider with one narrow notch-band,” Electron. Lett., vol. 53, pp. 1524-1526, Nov. 2017.

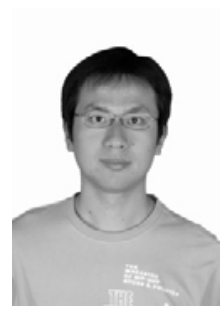

Feng Wei (M' 10) was born in Shaanxi Province, China, in 1978. He received the B. Eng. degree and M. Eng. degree in electrical engineering from Xidian University, Xi'an, China, in 2001 and 2004. From 2004 to 2006, he was a RF engineer in ZTE corporation. He received the $\mathrm{Ph} . \mathrm{D}$. degree in electrical engineering from Xidian University, Xi'an, China, in 2009. Since 2009, he has been with Collaborative Innovation Center of Information Sensing and Understanding at Xidian University and National Key Laboratory of Antennas and Microwave Technology, Xidian University, as a Lecturer and Associate Professor. From 2013 to 2014, he was a visiting scholar in Commonwealth Scientific and Industrial Research Organisation (CSIRO), Australia. He has authored or coauthored over 50 international and regional refereed journal papers. His recent research interests are mainly in the design of microwave components, circuits and RFID systems. He has served as a reviewer for IEEE TRANSACTIONS ON MICROWAVE THEORY AND TECHNIQUES and IEEE MICROWAVE AND WIRELESS COMPONENTS LETTERS.

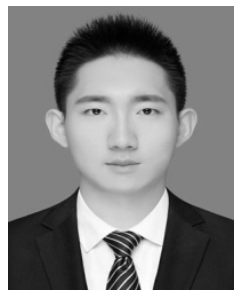

Zhi Jie Yang received the B. S. degree in electrica engineering from Xidian University, Xi'an, China, in 2016. He is currently working toward the M.S. degree in electrical engineering from Xidian University. His recent research interests are mainly in the design of balanced passive devices.

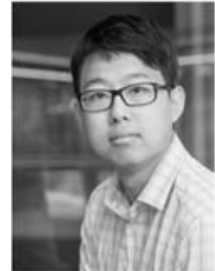

Pei-Yuan Qin was born in Liaoning Province, China, in 1983. He received a Bachelor Degree in Electronic Engineering from Xidian University, Xi'an, China, in 2006, and a joint Ph.D. Degree from Xidian University and Macquarie University, Australia, in electromagnetic fields and microwave technology in 2012.

From 2012 to 2015, he was a Postdoctoral Research Fellow in Commonwealth Scientific and Industrial Research Organisation (CSIRO), Australia. From 2015 to 2016, he was a Chancellor's Postdoctoral Research Fellow/Lecturer with University of Technology Sydney (UTS), Australia. Since 2017, he is a Senior Lecturer with UTS. His research interests are in the areas of reconfigurable antennas, phase shifters, reconfigurable reflectarrays, and MIMO communications.

Dr. Qin was a recipient of an Australia Research Council Discovery Early Career Researcher Award and also a recipient of the International Macquarie University Research Excellence Scholarship. He was awarded the Vice Chancellor's Commendation for academic excellence by Macquarie University. One of his papers was selected as 2016 Computer Simulation Technology University Publication Award. Since 2017, he has been serving as an Associate Editor of the IEEE Antennas and Wireless Propagation Letters.

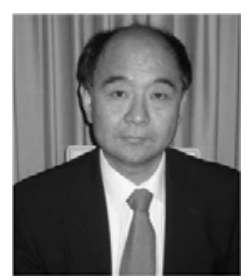

Y. Jay Guo (Fellow'2014) received a Bachelor Degree and a Master Degree from Xidian University in 1982 and 1984, respectively, and a PhD Degree from Xian Jiaotong University in 1987 , all in China. His research interest includes antennas, mm-wave and $\mathrm{THz}$ communications and sensing systems as well as big data technologies. He has published over 400 research papers and holds 24 patents in antennas and wireless systems. He is a Fellow of the Australian Academy of Engineering and Technology, a Fellow of IEEE and a Fellow of IET, and a member of the College of Experts of Australian Research Council (ARC). He has won a number of most prestigious Australian national awards, and was named one of the most influential engineers in Australia in 2014 and 2015.

Prof Guo is a Distinguished Professor and the founding Director of Global Big Data Technologies Centre at the University of Technology Sydney (UTS), Australia. Prior to this appointment in 2014, he served as a Director in CSIRO for over nine years, directing a number of ICT research portfolios. Before joining CSIRO, he held various senior technology leadership positions in Fujitsu, Siemens and NEC in the U.K.

Prof Guo has chaired numerous international conferences. He was the International Advisory Committee Chair of IEEE VTC2017, General Chair of ISAP2015, iWAT2014 and WPMC'2014, and TPC Chair of 2010 IEEE WCNC, and 2012 and 2007 IEEE ISCIT. He served as Guest Editor of special issues on "Antennas for Satellite Communications" and "Antennas and Propagation Aspects of 60-90GHz Wireless Communications," both in IEEE Transactions on Antennas and Propagation, Special Issue on "Communications Challenges and Dynamics for Unmanned Autonomous Vehicles," IEEE Journal on Selected Areas in Communications (JSAC), and Special Issue on "5G for Mission Critical Machine Communications”, IEEE Network Magazine.

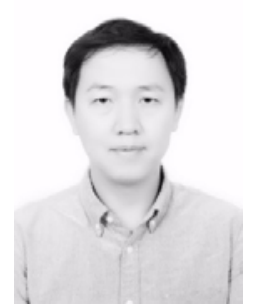

Bin Li was born in Tianjin, China. He received the B.Eng. degree from the Xidian University, Xi'an, China, in 2001, and the Ph.D. degree from City University of Hong Kong, in 2007, both in Electronic Engineering. He is currently an Associate Professor with the School of Information and Electronics, Beijing Institute of Technology, Beijing, China. In 2009, he was a Research Fellow in the State Key Laboratory of Millimeter Waves of City University of Hong Kong. Dr. Li has received Excellent Graduate Thesis in 2016 and 2018, respectively. His current research interests include Dielectric Resonator Antennas, Reflectarray/ Transmitarray, Millimeter-wave/THz antennas. Dr. Li has published more than 50 papers on domestic and international journals and conferences. The total citation number is more than 200. Dr $\mathrm{Li}$ is a communication reviewer of National Nature Science Foundation of China. He is also the peer reviewer of some international journals include IEEE Transactions on Antennas and Propagation, IEEE Antennas and Wireless Propagation Letters etc. 


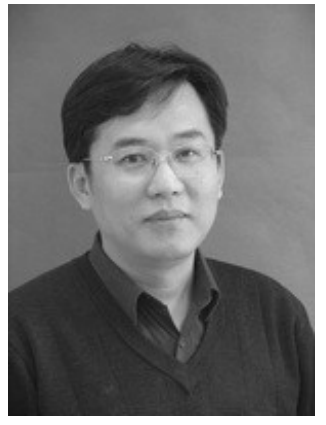

Xiao-Wei Shi received the B.Sc. degree in radio physics, and the $\mathrm{M}$. Eng. and $\mathrm{Ph}$. D. degrees, both in electrical engineering, all from Xidian University, Xi'an, China, in 1982, 1990, and 1995 , respectively. Since 1990 , he has been with the National Key Laboratory of Antennas and Microwave Technology, Xidian University, China, as a Lecturer, Associate Professor, and Professor. He is also Director of Office for Science \& Technology at Xidian University. He worked as a Postdoctoral Fellow at Electronics and Telecommunications Research Institute (ETRI) in Korea, from 1996 to 1997 . He has published over 50 international and regional refereed journal papers. His recent research interests are mainly in smart antennas design, electromagnetic inverse scattering, and electromagnetic compatibility. Dr. Shi received the first-class Prize of Excellent Teaching of Shaanxi Province in 1995 and Scientific Progress Awards of Shaanxi Province in 1992. 